Check for updates

Cite this: Chem. Commun., 2020, 56,6949

Received 3rd January 2020,

Accepted 11th May 2020

DOI: $10.1039 / \mathrm{c} 9 \mathrm{cc} 10071 \mathrm{~g}$

rsc.li/chemcomm

\section{A nitroreductase and glutathione responsive nanoplatform for integration of gene delivery and near-infrared fluorescence imaging $\dagger$}

\author{
Hong Liang, $\stackrel{\ddagger}{\ddagger}^{a}$ Qunjie Bi, $\ddagger^{a}$ Ao Hu, ${ }^{a}$ Xiaobing Chen, ${ }^{a}$ Rongrong Jin, ${ }^{a}$ Xu Song, ${ }^{\text {ab }}$ \\ Bowen Ke, (iD ${ }^{a}$ Matthias Barz (D) ${ }^{c}$ and $\mathrm{Yu}$ Nie (iD $* a$
}

\begin{abstract}
A novel platform rationally integrating indocyanine green analogues and an arginine-rich dendritic peptide with both nitroreductase (NTR) and glutathione (GSH) reduction responsive linkers was developed. This multifunctional platform can enable selective and efficient gene delivery and specific turn-on fluorescence imaging in tumors.
\end{abstract}

Tumor cells, tumor associated cells, and cellular and extracellular matrix components constitute an orchestrated "tumor microenvironment", promoting the growth, invasion and metastasis of tumors. ${ }^{1}$ A hallmark of solid tumor microenvironments is hypoxia, originating from an insufficient oxygen supply from the blood due to disorganized vasculature. ${ }^{2}$ Hence, the biomarkers of hypoxia, such as overexpressed reductive enzymes (including glutathione, nitroreductase, azoreductase and DT-diaphrose), ${ }^{3-5}$ could be useful for tumor diagnosis and antitumor treatment. ${ }^{6,7}$ Among them, NTR switchable fluorophores have received a lot of attention due to their exceptional sensitivity. ${ }^{8-11}$ A combination of fluorescence recovery with chemotherapy, photodynamic therapy and radiotherapy ${ }^{12,13}$ has achieved rapid development as a theranostic platform. However, tumor hypoxia is also known as the "Achilles' heel" of traditional photodynamic therapy (PDT), because severe tumor hypoxia hampers therapeutic outcomes of oxygendependent PDT and PDT potentiates hypoxia.

Gene therapy is a promising treatment which works by silencing abnormally overexpressed genes or compensating for defective genes, ${ }^{14,15}$ which has no conflict with the hypoxic conditions. This might be a good choice for the NTR triggered

\footnotetext{
${ }^{a}$ National Engineering Research Center for Biomaterials, Sichuan University, No. 29, Wangjiang Road, Chengdu 610064, P. R. China.

E-mail: nie_yu@scu.edu.cn,xusong2016@scu.edu.cn

${ }^{b}$ Institute of Regulatory Science for Medical Devices, National Engineering Research Center for Biomaterials, Sichuan University, No. 29, Wangjiang Road, Chengdu 610064, P. R. China

'Johannes Gutenberg-University Mainz, Organic Chemistry, Duesbergweg 10-14, MainZ, DE 55099, Germany

$\dagger$ Electronic supplementary information (ESI) available. See DOI: 10.1039/c9cc10071g

\$ The authors contributed equally to this article.
}

theranostic therapy. In particular, visual tracing of genes gives an intuitive and quantitative evaluation of the dynamic delivery processes at both the cellular and tissue levels in a real-time fashion. ${ }^{16,17}$ It is generally known that direct labeling of genes could influence their biological effect, the probes are usually physically encapsulated or chemically bonded to the periphery of the nanocarriers. However, these loading methods for fluorescent nanomaterials often suffer from premature leakage. ${ }^{18,19}$ Thus, an integrated fluorescent probe, consisting of borondipyrromethene (BODIPY) as the main component has been designed as a drug carrier, ${ }^{20}$ and PEGylated platinated-BODIPY molecules have been developed to combine chemical, photodynamic and photothermal therapies together for tumor ablation. $^{21}$ Our previous work has proved that arginine-rich amphiphilic lipopeptides exhibit excellent gene transfection activity, $^{22-24}$ and microenvironment-responsive modification could strongly promote both specificity and efficiency of gene expression. ${ }^{25,26}$ Our recent investigations further confirm that dual or multi-responsive triggers in carrier design provided a deeper biological understanding of the tumor micro-environment, and resulted in high-specific, highly efficient and low-toxic therapy. ${ }^{27,28}$ The high GSH concentration (at least 4 fold higher) is one of the important characteristics that distinguish tumor tissue/ cells from normal tissue/cells. ${ }^{29,30}$ Therefore, we intend to utilize the feature of high concentration of NTR and GSH around the tumor and intra-tumor cells simultaneously. Accordingly, a NTR-sensitive near-infrared fluorescent molecule was designed as the hydrophobic segment, whose fluorescence would be quenched due to photoinduced electron transfer. ${ }^{31-33}$

We herein developed NTR and GSH-induced "turn-on" assemblies (RNNS) for gene delivery and targeted imaging with real-time visualization of carrier metabolism (Scheme 1). The carriers possess an arginine-rich hydrophilic moiety for efficient gene condensation and cytomembrane penetration, a derivative of cyanine dye, in the skeleton of the amphiphilic molecule as the hydrophobic moiety for NTR-sensitive fluorescence imaging, and a disulfide bond as the trigger for GSH-responsive cargo release in tumor sites. 


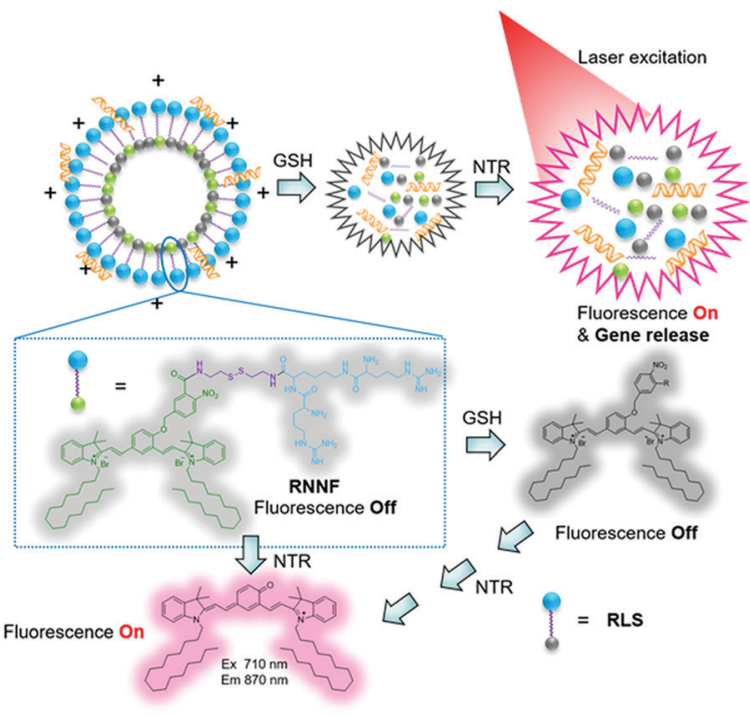

Scheme 1 The NTR and GSH responsive nanoplatform for gene delivery and fluorescence imaging.

The synthetic route to the target RNNF molecule and details are presented in Fig. S1 (ESI $\dagger$ ), and the structure characterization of each molecule has been performed through nuclear magnetic resonance spectroscopy (NMR) and MS (Fig. S2-S13, ESI $\dagger$ ). The amphiphilic RNNF molecule could self-assemble into nano-sized assemblies with a $Z$-average size of $340 \mathrm{~nm}$ and a zeta potential of $+29 \mathrm{mV}$. It could also be mixed with another amphiphilic molecule (RLS) synthesized in our previous work $^{23}$ at different molar ratios $(1 / 1,1 / 2$ and $1 / 3)$ to obtain smaller assemblies (RNNS, size from 130-185 nm) with better size distribution. RNNS-3 assemblies $(\mathrm{RNNF} / \mathrm{RLS}$, molar ratio = 1/3) showed a minimum size of $136 \mathrm{~nm}$ with a positive charge $(+22 \mathrm{mV}$ ) (Table S1, ESI $\dagger$ ). The larger diameter might be ascribed to both strong hydrophobicity and rigidity of the fluorescent molecule (RNNF) during self-assembly. ${ }^{34-36}$ While the addition of the flexible RLS molecule might be conducive to its tight integration with molecules, it results in smaller size of the assembly. ${ }^{23}$ The size of all assemblies increased after DNA condensation, and the zeta potential reduced slightly. As a representative, the morphology of RNNS-3 assemblies and RNNS-3/DNA complexes were observed through transmission electron microscopy (TEM). RNNS-3 assemblies showed a uniform discoid shape and RNNS-3/DNA complexes revealed a uniform ellipsoidal shape (Fig. 1A and B). The fluorescence spectral characteristics of various molecules and assemblies in organic or aqueous solutions were investigated to detect the on-demand "OFF" and "ON" signals, respectively (Fig. 1C). RNNF showed no fluorescence signal in organic solution, even after incubation with GSH. While the spectral peak significantly enhanced in the presence of both NTR and NADH. This indicated specific response of RNNF molecules to NTR with an outstanding turn-on effect on fluorescence recovery, which might be beneficial for reducing the background interference.

In order to evaluate the selective disassembly response of assemblies, RLS, RNNF, and RNNS-1, 2, 3 and 4 were incubated

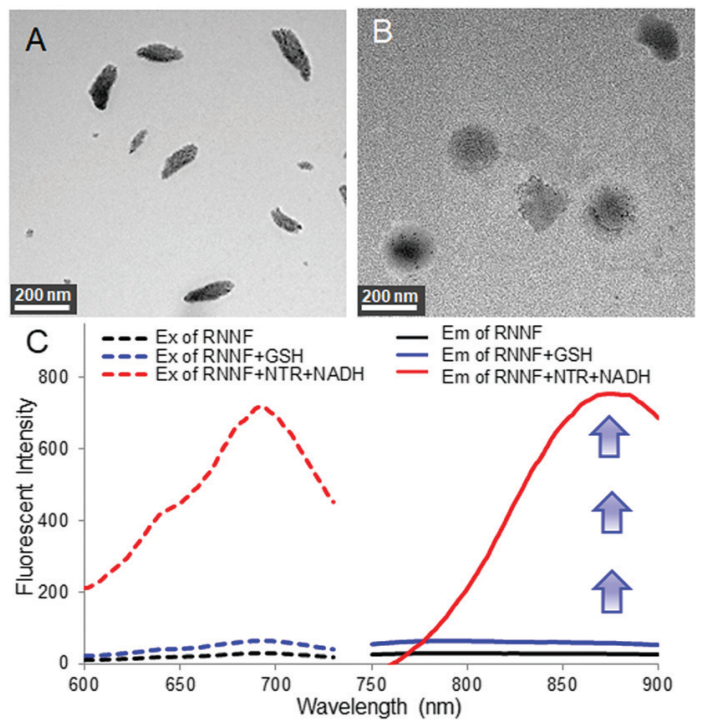

Fig. 1 Characterization of RNNS-3 assemblies and RNNS-3/DNA complexes by TEM and fluorescence spectra of RNNF in various solutions. TEM image of RNNS-3 assemblies (A) and RNNS-3/DNA complexes (B), respectively. (C) Fluorescence spectra of RNNF in various solutions with maximal excitation wavelength (dotted line, Ex $690 \mathrm{~nm}$ ) and emission wavelength (solid line, Em $870 \mathrm{~nm}$ ), respectively. Black lines: RNNF in methanol, red lines: RNNF in mixed solution (methanol/water, 1/1, v/v) with $10 \mathrm{mM}$ GSH for $2 \mathrm{~h}$, blue lines: RNNF in mixed solution (methanol/water, 1/1, v/v) with $5 \mu \mathrm{g} \mathrm{mL}^{-1} \mathrm{NTR}$ and $1 \mathrm{mM}$ $\mathrm{NADH}$ for $2 \mathrm{~h}$.

with GSH, NTR or their mixture, respectively. The changes in the size and zeta potential are shown in Fig. 2A-C and Fig. S14 (ESI $\dagger$ ). RLS and RNNF displayed the most rapid increase in size when incubated with GSH and NTR, respectively. While RNNF/RLS assemblies showed relatively slow responses in the presence of GSH or NTR. It might be that RNNF partly prevented the degradation of RLS by GSH, and RLS also disturbed the interaction between RNNF and NTR. What's more, noticeable
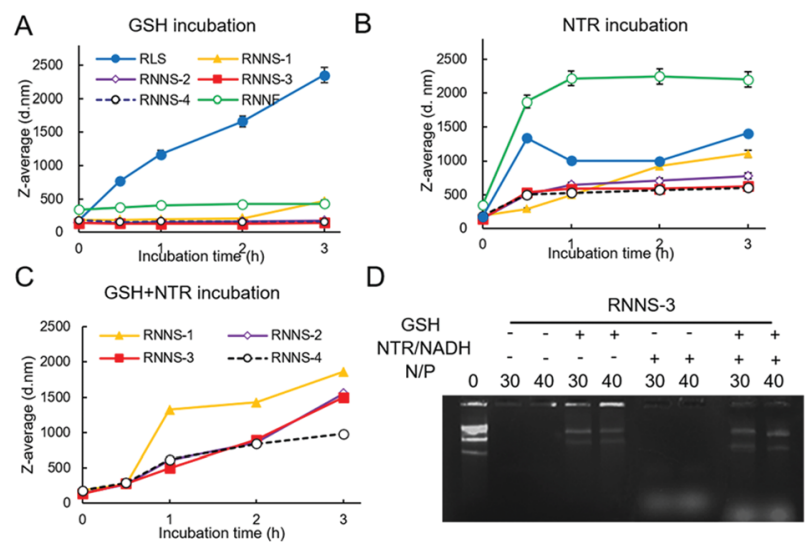

Fig. 2 The selective disassembly response and corresponding gene release of various assemblies in different conditions. The changes of size for various assemblies (RNNF, RLS and RNNS) after incubation with $10 \mathrm{mM}$ GSH (A), $5 \mu \mathrm{g} \mathrm{mL}{ }^{-1}$ NTR and $1 \mathrm{mM} \mathrm{NADH}$ (B), or mixture of GSH and NTR (C), respectively. Date are presented as means $\pm \mathrm{SD}(n=5)$. (D) The gene release ability of RNNS-3 in the presence of GSH, NTR or their mixture, respectively. 
changes in the size and zeta potential of all assemblies were observed in the presence of both GSH and NTR, revealing the successful disassembly.

Some research has found that assemblies with a rigid structure could be conducive to forming tight interaction with the cargo and improve their gene condensation ability. ${ }^{35,36}$ Thus, we evaluated the gene compaction ability of various assemblies with different N/P ratios through gel electrophoresis (Fig. S15A, ESI $\dagger$ ) and RNNS-3 was chosen as a representative for mixed assemblies. It was clear that pDNA could be completely dragged in the loading position by RLS at an N/P ratio of 60 , by RNNF at an $\mathrm{N} / \mathrm{P}$ ratio of 20 and by RNNS-3 at an N/P ratio of 30 , respectively. RNNF with a relatively rigid structure really showed benefit for the condensation, and its addition could contribute to gene compaction with lower N/P in the mixed assemblies. Consequently, gene release ability of these assemblies was investigated in the presence of GSH, NTR or their mixture, respectively (Fig. 2D and Fig. S15B, $\mathrm{ESI} \dagger$ ). The images showed that in the presence of either GSH or NTR alone, the release of gene in RNNF, RLS and RNNS assemblies was incomplete. This revealed that fluorescence could recover in NTR solution but without gene release (Fig. 1). However, all the assemblies could completely release the gene in the mixture of GSH and NTR.

Although it has been reported that the content of NTR is relatively high in tumor cells due to the hypoxic environment, ${ }^{37}$ there is hardly any relevant data to support this statement. ${ }^{8-11}$ Therefore, the content of NTR in tumor cells (HeLa) and nontumor cells (human umbilical vein endothelial cells, HUVEC) were detected using a Human NTR ELISA Kit, which indeed showed higher NTR content in HeLa cells than that in HUVEC (Fig. S16, ESI $\dagger$ ). In addition, GSH has also been confirmed to be highly expressed in HeLa cells. ${ }^{22,38}$ Subsequently fluorescence recovery of RNNS-3 gene complexes was studied in HeLa cells with HUVEC as a control (Fig. 3A). It was obviously found that the fluorescent signal in HeLa cells gradually enhanced with the incubation time, while only slight changes were observed in HUVEC. The semi-quantitative evaluation also confirmed these results, showing almost 5-fold difference in intensity (Fig. S17, ESI $\dagger$ ). We could speculate that the fluorescence of RNNS-3 could recover upon interaction with NTR and GSH in HeLa cells.

In vitro gene transfection activity of these assemblies was also studied on HeLa cells, and pEGFP plasmid acted as the model gene (Fig. 3B and Fig. S18, ESI $\dagger$ ). More green fluorescent spots were observed in the RNNS-3 group compared to the PEI or lipofectamine 2000 group, either in the presence or absence of $10 \%$ FBS. In the culture medium with $10 \%$ FBS, the gene transfection activity of these mixed assemblies decreased to a certain extent, but still far surpassed that of the control groups (Fig. 3B and Fig. S18, ESI $\dagger$ ). RNNS-3 assemblies showed the best gene transfection effect at $\mathrm{N} / \mathrm{P}=30$, reaching up to 30 -fold higher than commercial reagents (lipofectamine 2000 and PEI). Flow cytometry data also confirmed that RNNS-3 (N/P = 30) retained a high gene expression in HeLa cells with $10 \%$ serum (Fig. S19, ESI $\dagger$ ). The gene transfection efficiency would be gradually improved with an increase in the ratio of RNNF. But excessive rigidity would obstruct the interaction between the nanocarriers
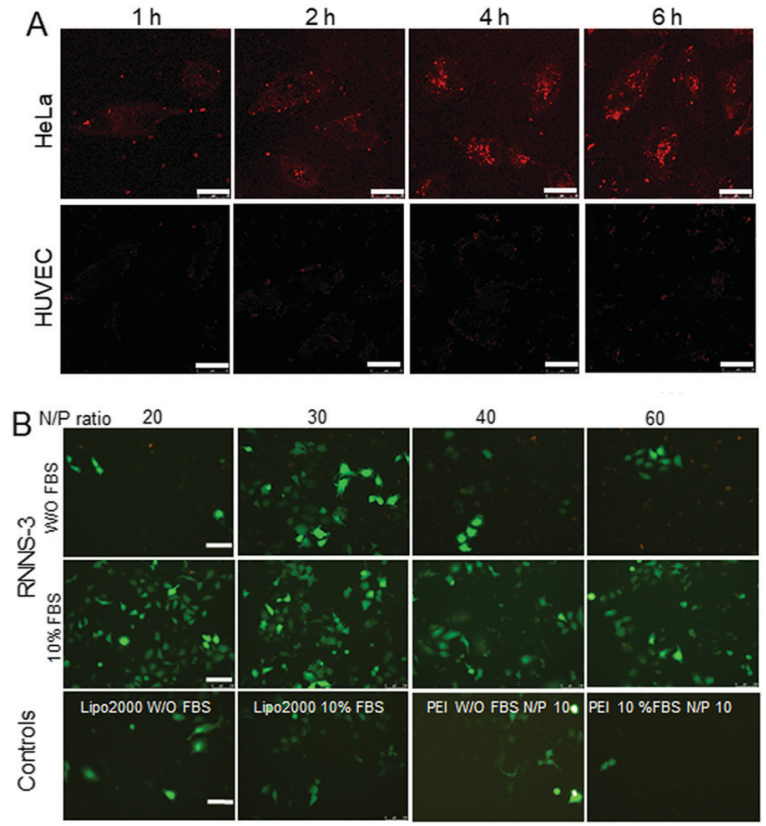

Fig. 3 Fluorescence recovery and in vitro gene transfection efficiency of RNNS-3. (A) Fluorescence recovery image of RNNS-3 observed through a laser scanning confocal microscope (CLSM). The plasmid DNA was condensed by RNNS-3 assemblies at N/P $=30$. HeLa (tumor cells) and HUVEC (non-tumor cells) were incubated with the RNNS-3/DNA complexes for different times (1, 2, 4 and $6 \mathrm{~h}$ ). The fluorescence signals (red) were observed under $663 \mathrm{~nm}$ laser-excitation and 700-800 nm emission signals. Scale bar $=100 \mu \mathrm{m}$. (B) Fluorescence microscopy images of HeLa cells with EGFP transfection for $48 \mathrm{~h}$ in the culture medium without or with $10 \%$ FBS. pEGFP plasmid DNA were condensed by RNNS-3 assemblies at different N/P ratios (N/P = 20, 30, 40 and 60). Lipofectamine 2000 and PEI $(M W=25000)$ acted as control groups. The N/P ratio of PEI/DNA complexes were $10 .^{22-24}$ Liposome 2000/DNA complex was $0.2 \mu \mathrm{L} / 100$ $\mathrm{ng}$ according to the protocol. Scale bar $=100 \mu \mathrm{m}$.

and cell membrane, and even hinder endocytosis and gene release. ${ }^{39}$ Meanwhile, cytotoxicity is another factor that influences the gene transfection effects, and might lead to the poor gene transfection efficiency of RNNS-1 (Fig. S20, ESI $\dagger$ ). The order of cytotoxicity for these assemblies was RNNS-1 > RNNS-2 > RNNS-3 > RNNS-4. Almost no toxicity was observed in RNNS-3 groups at concentrations below $60 \mu \mathrm{g} \mathrm{mL}^{-1}$, which was suitable for in vitro and in vivo applications. According to our previous works, RLS showed very low cytotoxicity. ${ }^{22-24}$ We speculated that cytotoxicity was mainly caused by RNNF. The addition of RLS has significant neutralizing effect on the rigidity of RNNF, thus reducing the cytotoxicity of mixed assemblies. As a result, the RNNS-3 assemblies with a partly rigid structure and low cytotoxicity show the best performance of gene transfection in culture medium with or without FBS.

In the animal experiment, RNNS-3/pEGFP showed a turn-on effect at the tumor site (Fig. S21, ESI $\dagger$ ). The near-infrared (NIR) strong fluorescence was maintained for $6 \mathrm{~h}$ post-injection, as shown in the living mice image (Fig. S21A, ESI $\dagger$ ) and ex vivo image of the isolated tissues (Fig. S21B, ESI $\dagger$ ). Images also reflected that the indocyanine green (ICG) analogs degraded from RNNS-3 could be metabolized by the liver and excreted through the kidneys. ${ }^{40}$ 


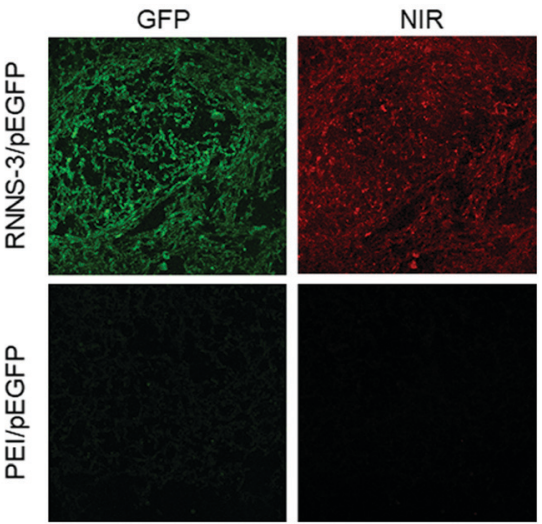

Fig. 4 In vivo green fluorescent protein expression and fluorescence signals in tumors $48 \mathrm{~h}$ after intratumoral injection of RNNS-3/pEGFP $(\mathrm{N} / \mathrm{P}=30)$ and $\mathrm{PEI} / \mathrm{pEGFP}(\mathrm{N} / \mathrm{P}=10)$.

This result confirmed that RNNS-3 could be degraded or metabolize and is safe for humans. In vivo gene transfection was studied $48 \mathrm{~h}$ post-injection (Fig. 4). Compared with the PEI/pEGFP group, the frozen sections of isolated tumors in the RNNS-3/pEGFP group showed considerable green fluorescence which agreed well with the result of the NIR channel, indicating that NIR provide the accurate location of gene expression in the tumor site.

In summary, we designed a type of amphiphilic lipopeptide molecule, RNNF, which could be used as a theranostic nanoplatform for cancer treatment through self-assembly. The mixed assemblies RNNS-3 (RNNF/RLS $=1 / 3$ ) have a minimum size of $136 \mathrm{~nm}$ and their fluorescence showed NTR-responsiveness with the maximum emission wavelength at $870 \mathrm{~nm}$. Although RNNF and RLS assemblies show different sensitive responsiveness to NTR and GSH respectively, RNNS-3 assemblies showed good responsiveness to both and good gene condensation and release ability in the presence of NTR or GSH. Moreover, with low cytotoxicity, RNNS-3 exhibited excellent fluorescence recovery and efficient gene transfection performance both in vitro and in vivo. Their gene transfection activity was even much better than commercial reagents such as lipofectamine 2000 and PEI (MW = 25 000).

This work was supported by the National Key Research and Development Plan of China (2017YFC1104601), the National Natural Science Foundation of China (NSFC, No. 81873921 and 51903174), the Sino-German Cooperation Group Project (GZ1512) and the Sichuan Science and Technology Program (2019JDJQ0027).

\section{Conflicts of interest}

There are no conflicts to declare.

\section{Notes and references}

1 D. Hanahan and R. A. Weinberg, Cell, 2011, 144, 646-674.

2 A. L. Harris, Nat. Rev. Cancer, 2002, 2, 38-47.

3 Y. Chen and L. Hu, Med. Res. Rev., 2009, 29, 29-64.
4 M. I. Uddin, S. M. Evans, J. R. Craft, L. J. Marnett, M. J. Uddin and A. Jayagopal, ACS Med. Chem. Lett., 2015, 6, 445-449.

5 W. J. Oyen, J. H. Kaanders and J. Bussink, Nucl. Med. Mol. Imaging, $2013,57,217-218$.

6 L. Zhang, X. Shan, L. Guo, J. Zhang, J. Ge, Q. Jiang and X. Ning, Analyst, 2018, 144, 284-289.

7 D. Ma, C. Huang, J. Zheng, W. Zhou, J. Tang, W. Chen, J. Li and R. Yang, Anal. Chem., 2019, 91, 1360-1367.

8 J. Zheng, Y. Shen, Z. Xu, Z. Yuan, Y. He, C. Wei, M. Er, J. Yin and H. Chen, Biosens. Bioelectron., 2018, 119, 141-148.

9 L. Cui, Y. Zhong, W. Zhu, Y. Xu, Q. Du, X. Wang, X. Qian and Y. Xiao, Org. Lett., 2011, 13, 928-931.

10 H. C. Huang, K. L. Wang, S. T. Huang, H. Y. Lin and C. M. Lin, Biosens. Bioelectron., 2011, 26, 3511-3516.

11 M. Srinivasarao, C. V. Galliford and P. S. Low, Nat. Rev. Drug Discovery, 2015, 14, 203-219.

12 Q. M. Zhang, C. Jin, J. H. Yu and W. Lu, ACS Omega, 2018, 3, 8813-8818.

13 Z. Liu, F. Song, W. Shi, G. Gurzadyan, H. Yin, B. Song, R. Liang and X. Peng, ACS Appl. Mater. Interfaces, 2019, 11, 15426-15435.

14 P. Zarogoulidis, E. Chatzaki, W. Hohenforst-Schmidt, E. P. Goldberg, G. Galaktidou, T. Kontakiotis, N. Karamanos and K. Zarogoulidis, Cancer Gene Ther., 2012, 19, 593-600.

15 X. M. Anguela and K. A. High, Annu. Rev. Med., 2019, 70, 273-288.

16 N. Boehnke, S. Correa, L. Hao, W. Wang, J. P. Straehla, S. N. Bhatia and P. Hammond, Angew. Chem., Int. Ed., 2020, 132, 2936.

17 X. He, Q. Luo, J. Zhang, P. Chen, H. J. Wang, K. Luo and X. Q. Yu, Nanoscale, 2019, 11, 12973-12982.

18 J. Croissant and J. I. Zink, J. Am. Chem. Soc., 2012, 134, 7628-7631.

19 D. Gyawali, S. Y. Zhou, R. T. Tran, Y. Zhang, C. Liu, X. C. Bai and J. Yang, Adv. Healthcare Mater., 2014, 3, 182-186.

20 X. G. Liu, M. Wu, Q. L. Hu, H. Z. Bai, S. Q. Zhang, Y. Q. Shen, G. P. Tang and Y. Ping, ACS Nano, 2016, 10, 11385-11396.

21 Z. Q. Guo, Y. L. Zou, H. He, J. M. Rao, S. S. Ji, X. N. Cui, H. T. Ke, Y. B. Deng, H. Yang, C. Y. Chen, Y. L. Zhao and H. B. Chen, Adv. Mater., 2016, 28, 10155-10164.

22 X. Chen, J. Yang, H. Liang, Q. Jiang, B. Ke and Y. Nie, J. Mater. Chem. $B, 2017,5,1482-1497$.

23 H. Liang, A. Hu, X. Chen, R. Jin, K. Wang, B. Ke and Y. Nie, J. Mater. Chem. B, 2019, 7, 915-926.

24 X. Xu, Q. Jiang, X. Zhang, Y. Nie, Z. Zhang, Y. Li, G. Cheng and Z. Gu, J. Mater. Chem. B, 2015, 3, 7006-7010.

25 Y. Y. He, G. Cheng, L. Xie, Y. Nie, B. He and Z. W. Gu, Biomaterials, 2013, 34, 1235-1245.

26 Y. Y. He, Y. Nie, L. Xie, H. M. Song and Z. W. Gu, Biomaterials, 2014, 35, 1657-1666.

27 X. Jia, Y. Zhang, Y. Zou, Y. Wang, D. Niu, Q. He, Z. Huang, W. Zhu, H. Tian, J. Shi and Y. Li, Adv. Mater., 2018, 30, e1704490.

28 W. Tang, W. Fan, Z. Wang, W. Zhang, S. Zhou, Y. Liu, Z. Yang, E. Shao, G. Zhang, O. Jacobson, L. Shan, R. Tian, S. Cheng, L. Lin, Y. Dai, Z. Shen, G. Niu, J. Xie and X. Chen, ACS Nano, 2018, 12, 12269-12283.

29 P. Kuppusamy, H. Li, G. Ilangovan, A. J. Cardounel, J. L. Zweier, K. Yamada, M. C. Krishna and J. B. Mitchell, Cancer Res., 2002, 62, 307-312.

30 R. Mo and Z. Gu, Mater. Today, 2016, 19, 274-283.

31 A. Chevalier, Y. Zhang, O. M. Khdour, J. B. Kaye and S. M. Hecht, J. Am. Chem. Soc., 2016, 138, 12009-12012.

32 S. Y. Lim, K. H. Hong, D. I. Kim, H. Kwon and H. J. Kim, J. Am. Chem. Soc., 2014, 136, 7018-7025.

33 L. Feng, R. Xie, C. Wang, S. Gai, F. He, D. Yang, P. Yang and J. Lin, ACS Nano, 2018, 12, 11000-11012.

34 M. Piest and J. F. J. Engbersen, J. Controlled Release, 2010, 148, 83-90.

35 B. Wang, R. M. Zhao, J. Zhang, Y. H. Liu, Z. Huang, Q. Y. Yu and X. Q. Yu, Eur. J. Med. Chem., 2017, 136, 585-595.

36 S. Raffy and J. Teissié, Biophys. J., 1999, 76, 2072-2080.

37 Z. Zhou, J. Song, L. Nie and X. Chen, Chem. Soc. Rev., 2016, 45, 6597-6626.

38 L. Y. Niu, Y. S. Guan, Y. Z. Chen, L. Z. Wu, C. H. Tung and Q. Z. Yang, J. Am. Chem. Soc., 2012, 134, 18928-18931.

39 C. L. Grigsby and K. W. Leong, J. R. Soc., Interface, 2010, 7, 67-82.

40 J. Zhao, Z. Wan, C. Zhou, Q. Yang, J. Dong, X. Song and T. Gong, Pharm. Res., 2018, 35, 196. 